\title{
BMJ Open Effect of the adjuvanted (AS03) A/H1N1 2009 pandemic influenza vaccine on the risk of rejection in solid organ transplant recipients in England: a self-controlled case series
}

Catherine Cohet, ${ }^{1}$ François Haguinet, ${ }^{1}$ Gaël Dos Santos, ${ }^{2}$ Dave Webb, ${ }^{3}$ John Logie, ${ }^{3}$ Germano LC Ferreira, ${ }^{1}$ Dominique Rosillon, ${ }^{1}$ Vivek Shinde ${ }^{1}$

To cite: Cohet $C$, Haguinet $F$, Dos Santos G, et al. Effect of the adjuvanted (ASO3) A/ H1N1 2009 pandemic influenza vaccine on the risk of rejection in solid organ transplant recipients in England: a self-controlled case series. BMJ Open 2016;6:e009264. doi:10.1136/bmjopen-2015009264

- Prepublication history and additional material is available. To view please visit the journal (http://dx.doi.org/ 10.1136/bmjopen-2015009264).

Received 30 June 2015 Revised 22 September 2015 Accepted 20 October 2015

CrossMark

For numbered affiliations see end of article.

Correspondence to Dr Catherine Cohet; catherine.x.cohet@gsk.com

\section{ABSTRACT}

Objective: To assess the risk of solid organ transplant (SOT) rejection after vaccination with the adjuvanted (AS03) A/H1N1 2009 pandemic influenza vaccine Pandemrix.

Design: Self-controlled case series (SCCS) in the UK Clinical Practice Research Datalink (CPRD) and its linked component of the Hospital Episodes Statistics (HES) inpatient database. Analyses were conducted using the SCCS method for censored, perturbed or curtailed post-event exposure.

Participants: Of the 184 transplant recipients having experienced at least one SOT rejection (liver, kidney, lung, heart or pancreas) during the study period from 1 October 2009 to 31 October 2010, 91 participants were included in the main analysis, of which 71 had been exposed to Pandemrix.

Main outcome measures: Occurrence of SOT rejection during risk (30 and 60 days after any Pandemrix dose) and control periods. Covariates in the CPRD included time since transplantation, seasonal influenza vaccination, bacterial and viral infections, previous SOT rejections and malignancies.

Results: The relative incidence (RI) of rejection of any one of the five transplanted organs, adjusted for time since transplantation, was $1.05(95 \% \mathrm{Cl} 0.52$ to 2.14$)$ and $0.80(95 \% \mathrm{Cl} 0.42$ to 1.50$)$ within 30 and 60 days after vaccination, respectively. Similar estimates were observed for rejection of a kidney only, the most commonly transplanted organ (RI within 30 days after vaccination: $0.85(95 \% \mathrm{Cl} 0.38$ to 1.90)). Across various models and sensitivity analyses, RI estimates remained stable and within a consistent range around 1.0 .

Conclusions: These results suggest a reassuring safety profile for Pandemrix with regard to the risk of rejection in SOT recipients in England and contribute to inform the benefit-risk of AS03adjuvanted pandemic influenza vaccines in transplanted patients in the event of future pandemics.

Trial registration number: NCT01715792.
Strengths and limitations of this study

- First pharmacoepidemiological analysis to formally assess the risk of solid organ transplant rejection following vaccination with an adjuvanted H1N1 2009 pandemic influenza vaccine.

- The data set combines vaccine exposure and covariate data from the Clinical Practice Research Datalink (CPRD) and linked clinical outcome data from the Hospital Episodes Statistics (HES) database, maximising the likelihood of capturing and characterising transplant rejection cases.

- The analysis uses the self-controlled case series for perturbed post-event exposure that handles fixed measured and unmeasured confounders while taking into account that the occurrence of rejection might modify the probability of being vaccinated.

- Availability or completeness of covariate data for rejection risk factors other than vaccination is limited in the CPRD.

- Other limitations include uncertainty on the duration of the risk period and limited generalisability to solid organs other than the kidney.

\section{INTRODUCTION}

Solid organ transplant (SOT) rejection is a major cause of graft failure in transplanted patients. Owing to the increased risk of complications associated with the influenza virus infection, transplant recipients are a high-risk group recommended for influenza vaccination. ${ }^{1-4}$ Following the WHO's declaration of a pandemic in June 2009, vaccines against pandemic influenza $\mathrm{A} / \mathrm{H} 1 \mathrm{~N} 1$ were developed, with specific pharmacovigilance monitoring implemented by vaccine manufacturers. Spontaneous cases of SOT rejection in temporal association with GSK Vaccines' inactivated monovalent AS03-adjuvanted A/H1N1 2009 influenza vaccines Pandemrix and Arepanrix ${ }^{\mathrm{TM}}$ (registered 
trademarks of the GSK group of companies) were reported in Europe and Canada, respectively. The majority of these case reports described the presence of risk factors (eg, noncompliance with immunosuppressive regimen, acute infection, cyclosporine nephropathy, prior rejection episodes) that may have caused or contributed to the rejection..$^{5-7}$ Nonetheless, given that transplanted patients will remain a high-risk priority group for immunisation with any future pandemic influenza vaccine, the European Medicines Agency (EMA) requested an evaluation of this safety signal, including the present study.

Despite theoretical concerns that vaccination could trigger rejection through induction of antihuman leucocyte antigen (HLA) antibodies, ${ }^{8}$ most studies evaluating the effect of seasonal influenza vaccination on the risk of SOT rejection have shown no increased risk of allograft dysfunction or clinical rejection. ${ }^{129-14}$ With regard to the safety of pandemic H1N1 influenza vaccination in transplanted patients, data are more scarce. The majority of published studies are descriptive and simultaneously evaluate immunogenicity and the safety profile of the vaccines in small case series. In most studies, there is no increased risk of allograft dysfunction or clinical rejection. ${ }^{2}$ 15-25 However, some studies describe increases in histological cellular rejection or de novo detection of anti-HLA antibodies, with or without clinical rejection or change in graft function. ${ }^{5-7}$ Recent reviews of published studies of both seasonal and pandemic influenza vaccination of transplanted patients conclude that there is clinical evidence that influenza infection is more likely than vaccination to be associated with a risk of allograft dysfunction. Despite some evidence of transiently increased cellular alloreactivity, influenza vaccination (pandemic and seasonal) is considered to have an acceptable safety profile in transplanted patients. ${ }^{1} 223$

To the best of our knowledge, there has been no previous pharmacoepidemiology study assessing the risk of SOT rejection following vaccination with a $2009 \mathrm{~A} / \mathrm{H} 1 \mathrm{~N} 1$ pandemic influenza vaccine. This Post-Authorisation Safety Study (PASS) was therefore designed to conduct a formal retrospective investigation of the association between Pandemrix and SOT rejection. The study was conducted in the UK, where Pandemrix was the primary vaccine administered during the 2009 influenza pandemic. $^{2627}$

\section{METHODS}

Settings

The study was based on the population registered in the UK Clinical Practice Research Datalink (CPRD), a nationally representative longitudinal coded anonymised medical database containing data from approximately 680 primary care practices, and linked data from hospital inpatient care. ${ }^{28-30}$ Since it was assumed that the large majority of transplanted patients are followed in specialty hospital settings and that a proportion of SOT rejection events might not be recorded in the CPRD primary care data, cases were identified via the linkage between the CPRD and the Hospital Episodes Statistics (HES) inpatient database, which contains patient details and discharge diagnoses for all admissions to National Health Service (NHS) hospitals in England (approximately $60 \%$ of the CPRD population are eligible for HES linkage). ${ }^{31}{ }^{32}$ The 2012-Q3 release of the CPRD was used.

\section{Vaccine exposure}

The exposure of interest was vaccination with Pandemrix, GSK Vaccines' antigen-sparing A(H1N1)pmd09 splitvirion inactivated vaccine adjuvanted with an Adjuvant System containing $\alpha$-Tocopherol and squalene in an oil-in-water emulsion (AS03). According to data from the UK Department of Health based on the ImmForm national survey, Pandemrix was used widely and in the majority of targeted groups, with $>99 \%$ of immunised individuals having received this vaccine. ${ }^{33}{ }^{34}$ Vaccination with a seasonal trivalent influenza vaccine (TIV), a potential confounder in the association between Pandemrix and SOT rejection, was also assessed. The occurrence and timing of pandemic and seasonal TIV vaccinations were retrieved from the CPRD.

\section{Study population}

Eligible participants were SOT recipients with at least one rejection of a transplanted heart, lung, kidney, liver or pancreas recorded in the HES inpatient database between 1 October 2009 and 31 October 2010, and considered acceptable for research in the CPRD (ie, with no break in their records, and with information available on year of birth, registration date and gender)..$^{30}$ Participants also had to have an HES and CPRD follow-up covering the 180 -day period preceding 1 October 2009 (ie, back to 4 April 2009) to contribute to the analyses, with no interruption in their permanent CPRD registration between 4 April 2009 and the end of follow-up (ie, 31 October 2010, death or withdrawal from the database).

\section{Study design}

We used the self-controlled case series (SCCS) method, which relies on the within-person comparison of occurrence of outcomes of interest during risk and control periods, ${ }^{35} 36$ thereby inherently controlling for fixed measured and unmeasured confounding factors, including confounding by indication. The choice of the risk periods was based on the latency period observed in spontaneous case reports; on the hypothesised risk period following known exposures such as infections; and on experience from post-vaccination risk windows for adverse events following immunisation. The primary objective was to assess the risk of rejection within 30 days after vaccination with Pandemrix. In the absence of strong supportive evidence for the duration of the risk period, a 60-day period was also considered in sensitivity 
analyses. The study period ranged from 1 October 2009 to 31 October 2010, to include the H1N1 mass immunisation campaign and to allow sufficient follow-up time for participants vaccinated late in the campaign. Individual observation periods began either on 1 October 2009 or at the time of transplantation if it occurred after 1 October 2009, and ended on 31 October 2010 or at a new transplantation event. The control periods corresponded to the observation period excluding the risk periods (figure 1).

Case-finding algorithms in the HES and the CPRD were developed to identify events (transplantations and rejections) and covariates of interest such as risk factors for SOT rejection. Transplantations were detected in the HES and the CPRD: each transplantation record in the HES (OPCS procedure code) was considered a distinct event; any transplantation code recorded in the CPRD for the same participant within 14 days before or after the HES event was considered the same transplantation event; and a CPRD code constituted a distinct transplantation event if it was separated by more than 14 days from any other transplantation record in the HES or the CPRD. Records of SOT rejections were identified in the HES only, through ICD10 diagnosis codes and OPCS procedure codes: records were considered as different events if they were separated by more than 30 days or in case of a new transplantation record between rejection events. Vaccinations with Pandemrix or with a TIV were identified in the CPRD by means of READ, immunisation and Product codes. Covariates of interest were detected in the CPRD by means of READ codes.

\section{Statistical analyses}

We applied the SCCS method for censored, perturbed or curtailed post-event exposure, ${ }^{37}$ which relies on the assumption that the occurrence of an event-here, SOT rejection-might modify a subsequent exposure-here, the probability of being vaccinated. Under such a scenario, only the first rejection was considered for each participant, that is, individual observation periods were censored at any subsequent rejection event.
Estimates of the relative incidence (RI) of SOT rejection were derived within 30 and 60 days following vaccination, as compared with the control period. A preliminary feasibility assessment in the CPRD revealed that the risk of SOT rejection consistently decreased with increasing time since transplantation (data not shown). Therefore, time since transplantation was included in all models by categorising the baseline risk after transplantation into four periods: 0-30, 31-90, 91-180 and $>180$ days. Participants not exposed to Pandemrix during the observation period were included in the analyses to better control for time since transplantation and other risk factor covariates.

Using the modified SCCS method, the RI was estimated using unbiased estimating equations. ${ }^{37}$ A Statistical Analysis Software (SAS) program was developed to derive the sandwich variance estimator from the estimating equations in settings with multiple exposures and covariates, given that the $95 \%$ CI could not be computed using the nonparametric bootstrapping, ${ }^{37}$ as (1) the rate of convergence among bootstrap samples was low for some analyses and (2) part of the bootstrap samples did not include covariates for which all participants were in the same category. A sandwich variance estimator was used to obtain the Wald CI of the RI. RI estimates were further adjusted in models including covariates, provided covariate information was available. Assumptions were made to define the risk period for each covariate: 30 days for vaccination with a TIV, and for respiratory, opportunistic and acute bacterial infections; and 365 days for chronic infections and malignancies.

The standard SCCS method ${ }^{38}$ was used as a sensitivity analysis. An exploratory analysis restricted to participants with no previous rejection during the 180 days prior to the study period was also performed to evaluate the effect of a previous rejection on the risk of a subsequent rejection. Analyses were also performed by a transplanted organ, if a minimum of 10 rejection events were observed for a given organ. Analyses were performed using SAS V.9.2 (SAS Institute, Cary, North Carolina, USA). ${ }^{39}$

\section{Figure 1 Study design.}

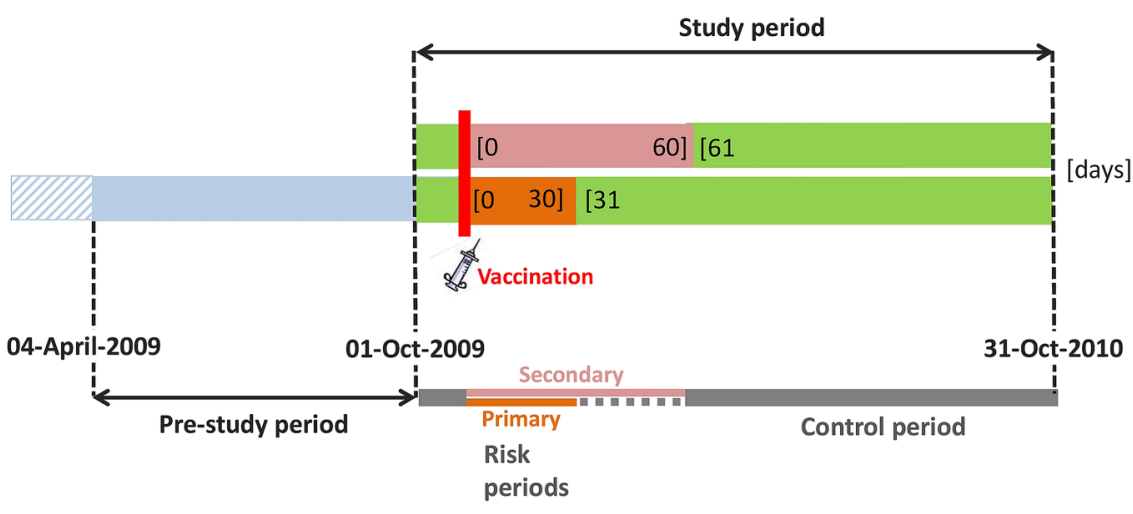

Data collection 


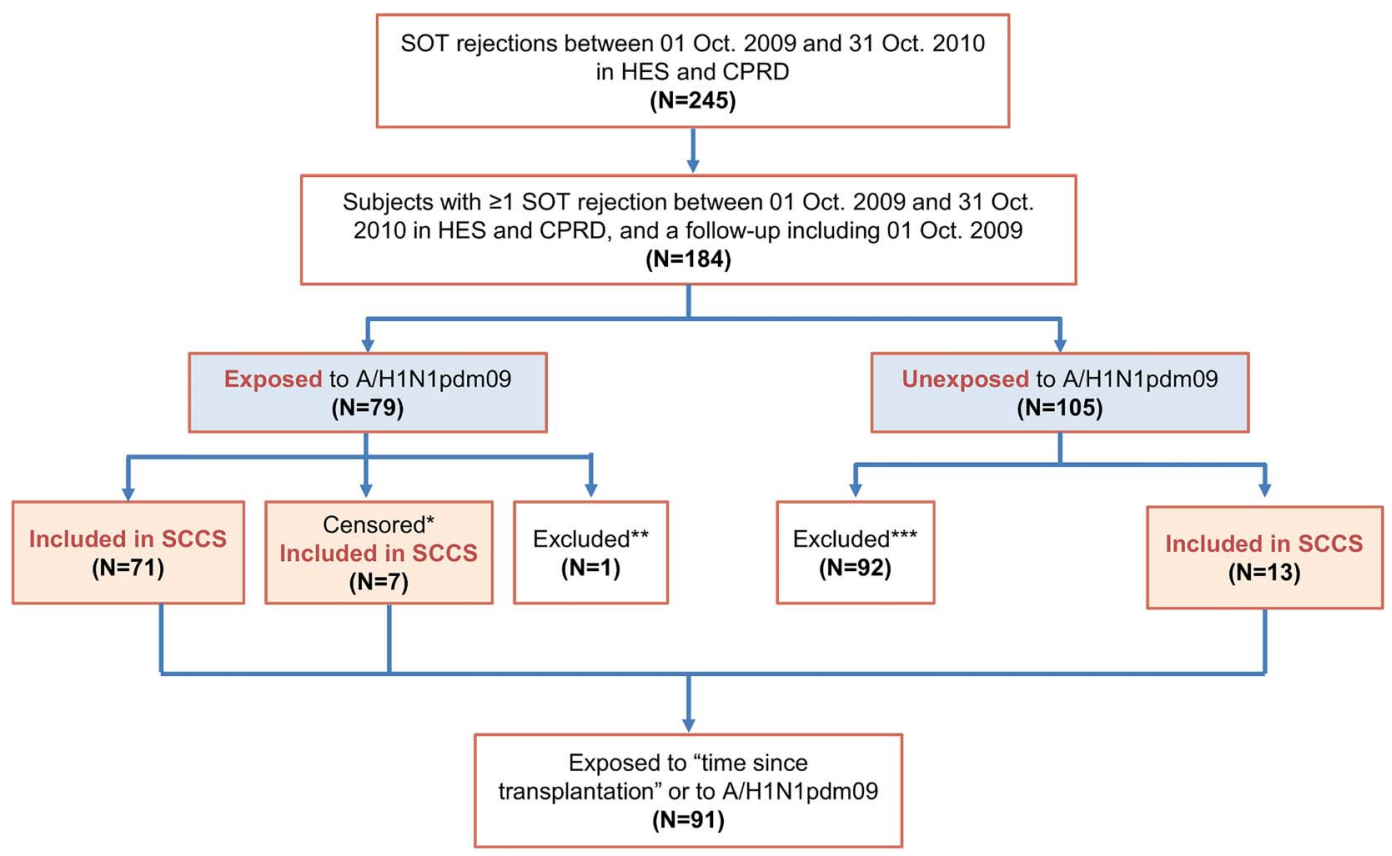

Figure 2 Disposition of study subjects. SOT, solid organ transplant; HES, Hospital Episodes Statistics database; CPRD, Clinical Practice Research Datalink; SCCS, self-controlled case series; A/H1N1pdm09, AS03 adjuvanted A/California/7/2009 like strain vaccine; $\mathrm{N}$, number of participants. *Transplantation after vaccination: $\mathrm{N}=6$ (including one with transplantation and rejection on same day); second rejection before vaccination $(\mathrm{N}=1)$; included in SCCS as unexposed. ${ }^{* *}$ Second transplantation (after rejection) before vaccination $(\mathrm{N}=1)$. ${ }^{* * *}$ Transplantation occurred before 4 April 2009, that is, before the 180 days preceding the beginning of the study period (see figure 1).

\section{RESULTS}

\section{Description of the study population}

Between 1 October 2009 and 31 October 2010, a total of 245 SOT rejection events were reported in HES; from these, 184 unique participants with follow-up including 1 October 2009 were identified, and of these, 79 had been exposed to Pandemrix. The study data set included 71 exposed and 20 unexposed patients (figure 2). The latter had a time period since transplantation lower than 180 days, and were included in the models to better account for time since transplantation, as described in the Methods section.

Since the SCCS method measures risk in exposed cases only, it is important to assess the differences between exposed and unexposed participants, that is, would the observed effect be similar in the unexposed group if the participants had been exposed? Table 1 shows the distribution of the main characteristics of the 79 exposed and 105 unexposed participants. Overall, the mean age was 50.2 years, $45.7 \%$ participants were female, and $5.4 \%$ died from any cause during the follow-up. Twenty-three participants had at least one rejection between 4 April 2009 and 1 October 2010. A record of transplantation during this period was available for $36 / 184$ patients (19.6\%), and kidney was the most frequently transplanted organ. Although this patient population is at high risk of infectious diseases, only a small proportion had records of infections. The two groups did not differ with regard to covariates of interest.
One and two doses of Pandemrix were administered to $62 / 79$ and $17 / 79$ exposed patients, respectively. A total of 73 patients received both a TIV and Pandemrix: in the 2009-2010 season only $(\mathrm{N}=42)$; in the 2009-2010 and early 2010-2011 seasons $(\mathrm{N}=29)$; and in the early 20102011 season only $(\mathrm{N}=2)$. Twenty-eight participants received a TIV within 4 weeks of a Pandemrix dose administration. Figure 3 shows the distribution of rejection events and vaccinations throughout the study period: the distribution of rejections appeared to remain stable over time. The majority of vaccine doses were administered between October 2009 and January 2010 , consistent with the timing of the UK mass immunisation campaign. ${ }^{27}$

\section{Risk estimates}

RI estimates for SOT rejection associated with Pandemrix, adjusted for time since transplantation, were $1.05(95 \%$ CI 0.52 to 2.14 ) and 0.80 (95\% CI 0.42 to 1.50 ) within 30 and 60 days after vaccination, respectively (figure 4). When covariates with available information (vaccination with a TIV, opportunistic infections and malignancies/ cancers) were included in the model, the RI within 30 and 60 days after Pandemrix vaccination was $1.29(95 \%$ CI 0.63 to 2.63) and 0.91 (95\% CI 0.49 to 1.70$)$, respectively.

In sensitivity analyses, we used the standard SCCS method that does not account for perturbed post-event exposure and thus included participants with SOT rejections subsequent to the rejection event captured in the 
Table 1 Main characteristics of participants with SOT rejection during the study period (1 October 2009 to 31 October 2010)

\begin{tabular}{|c|c|c|c|}
\hline Characteristics & $\begin{array}{l}\text { Exposed participants } \\
\mathrm{N}=79 \\
\text { Value } \\
\text { or } \mathrm{n}(\%)\end{array}$ & $\begin{array}{l}\text { Unexposed participants } \\
\mathrm{N}=105 \\
\text { Value } \\
\text { or } \mathrm{n}(\%)\end{array}$ & $\begin{array}{l}\text { Total } \\
\mathrm{N}=184 \\
\text { Value } \\
\text { or } \mathrm{n}(\%)\end{array}$ \\
\hline \multicolumn{4}{|l|}{ Age at 1 October 2009} \\
\hline $\begin{array}{l}\text { Mean age (years) } \pm S D \\
\text { (minimum; maximum) }\end{array}$ & $\begin{array}{l}51.3 \pm 14.54 \\
(9 ; 91)\end{array}$ & $\begin{array}{l}49.3 \pm 21.58 \\
(1 ; 89)\end{array}$ & $\begin{array}{l}50.2 \pm 18.86 \\
(1 ; 91)\end{array}$ \\
\hline \multicolumn{4}{|l|}{ Age group at 1 October 2009 (years) } \\
\hline$(0-17)$ & $3(3.8)$ & $9(8.6)$ & $12(6.5)$ \\
\hline$(18-44)$ & $20(25.3)$ & $33(31.4)$ & $53(28.8)$ \\
\hline$(45-60)$ & $32(40.5)$ & $31(29.5)$ & $63(34.2)$ \\
\hline $61+$ & $24(30.4)$ & $32(30.5)$ & $56(30.4)$ \\
\hline \multicolumn{4}{|l|}{ Gender } \\
\hline Female & $33(41.8)$ & $51(48.6)$ & $84(45.7)$ \\
\hline Male & $46(58.2)$ & $54(51.4)$ & $100(54.3)$ \\
\hline \multicolumn{4}{|c|}{ Number of rejections between 4 April 2009 and 1 October 2009} \\
\hline None & $70(88.6)$ & $91(86.7)$ & $161(87.5)$ \\
\hline At least one & $9(11.4)$ & $14(13.3)$ & $23(12.5)$ \\
\hline \multicolumn{4}{|c|}{ Organs transplanted before or during the study period* } \\
\hline Heart & $1(1.3)$ & $0(0.0)$ & $1(0.5)$ \\
\hline Kidney & $9(11.4)$ & $10(9.5)$ & $19(10.3)$ \\
\hline Kidney and liver & $0(0.0)$ & $2(1.9)$ & $2(1.1)$ \\
\hline Kidney and pancreas & $2(2.5)$ & $0(0.0)$ & $2(1.1)$ \\
\hline Liver & $3(3.8)$ & $6(5.7)$ & 9 (4.9) \\
\hline Lung & $2(2.5)$ & $0(0.0)$ & $2(1.1)$ \\
\hline Pancreas & $1(1.3)$ & $0(0.0)$ & $1(0.5)$ \\
\hline No transplantation recorded & $61(77.2)$ & $87(82.9)$ & $148(80.4)$ \\
\hline \multicolumn{4}{|c|}{ Organs rejected before or during the study period* } \\
\hline Heart & $4(5.1)$ & $4(3.8)$ & $8(4.3)$ \\
\hline Kidney & $56(70.9)$ & $39(37.1)$ & $95(51.6)$ \\
\hline Kidney and liver & $0(0.0)$ & $1(1.0)$ & $1(0.5)$ \\
\hline Kidney and pancreas & $1(1.3)$ & $0(0.0)$ & $1(0.5)$ \\
\hline Liver & $7(8.9)$ & $11(10.5)$ & $18(9.8)$ \\
\hline Lung & $4(5.1)$ & $1(1.0)$ & $5(2.7)$ \\
\hline Unspecified & $7(8.9)$ & $49(46.7)$ & $56(30.4)$ \\
\hline \multicolumn{4}{|c|}{ Number of transplantations between 1 October 2009 and 31 October 2010} \\
\hline 0 & $67(84.8)$ & $89(84.8)$ & $156(84.8)$ \\
\hline 1 & $11(13.9)$ & $13(12.4)$ & $24(13.0)$ \\
\hline 2 & $1(1.3)$ & $2(1.9)$ & $3(1.6)$ \\
\hline 4 & $0(0.0)$ & $1(1.0)$ & $1(0.5)$ \\
\hline \multicolumn{4}{|c|}{ Respiratory infection(s) between 1 September 2009 and 31 October 2010} \\
\hline No & $77(97.5)$ & $105(100)$ & $182(98.9)$ \\
\hline Yes & $2(2.5)$ & $0(0.0)$ & $2(1.1)$ \\
\hline \multicolumn{4}{|c|}{ Acute bacterial infection(s) between 1 September 2009 and 31 October 2010} \\
\hline No & $79(100.0)$ & $105(100.0)$ & $184(100.0)$ \\
\hline Yes & $0(0.0)$ & $0(0.0)$ & $0(0.0)$ \\
\hline \multicolumn{4}{|c|}{ Opportunistic infection(s) between 1 September 2009 and 31 October 2010} \\
\hline No & $73(92.4)$ & $103(98.1)$ & $176(95.7)$ \\
\hline Yes & $6(7.6)$ & $2(1.9)$ & $8(4.3)$ \\
\hline \multicolumn{4}{|c|}{ Chronic viral infection(s) between 1 October 2008 and 31 October 2010} \\
\hline No & $79(100.0)$ & $105(100.0)$ & $184(100.0)$ \\
\hline Yes & $0(0.0)$ & $0(0.0)$ & $0(0.0)$ \\
\hline \multicolumn{4}{|c|}{ Malignancy/cancer(s) between 01 October 2008 and 31 October 2010} \\
\hline No & $70(88.6)$ & $99(94.3)$ & $169(91.8)$ \\
\hline Yes & $9(11.4)$ & $6(5.7)$ & $15(8.2)$ \\
\hline \multicolumn{4}{|l|}{ Reasons for end of follow-up } \\
\hline End of study period (31 October 2010) & $74(93.7)$ & $94(89.5)$ & $168(91.3)$ \\
\hline Death & $4(5.1)$ & $6(5.7)$ & $10(5.4)$ \\
\hline End of CRPD follow-up & $1(1.3)$ & $5(4.8)$ & $6(3.3)$ \\
\hline
\end{tabular}


Figure 3 Biweekly frequency of Pandemrix vaccination and of SOT rejection events during the 31 October 2010). SOT, solid organ transplant. study period (1 October 2009 to

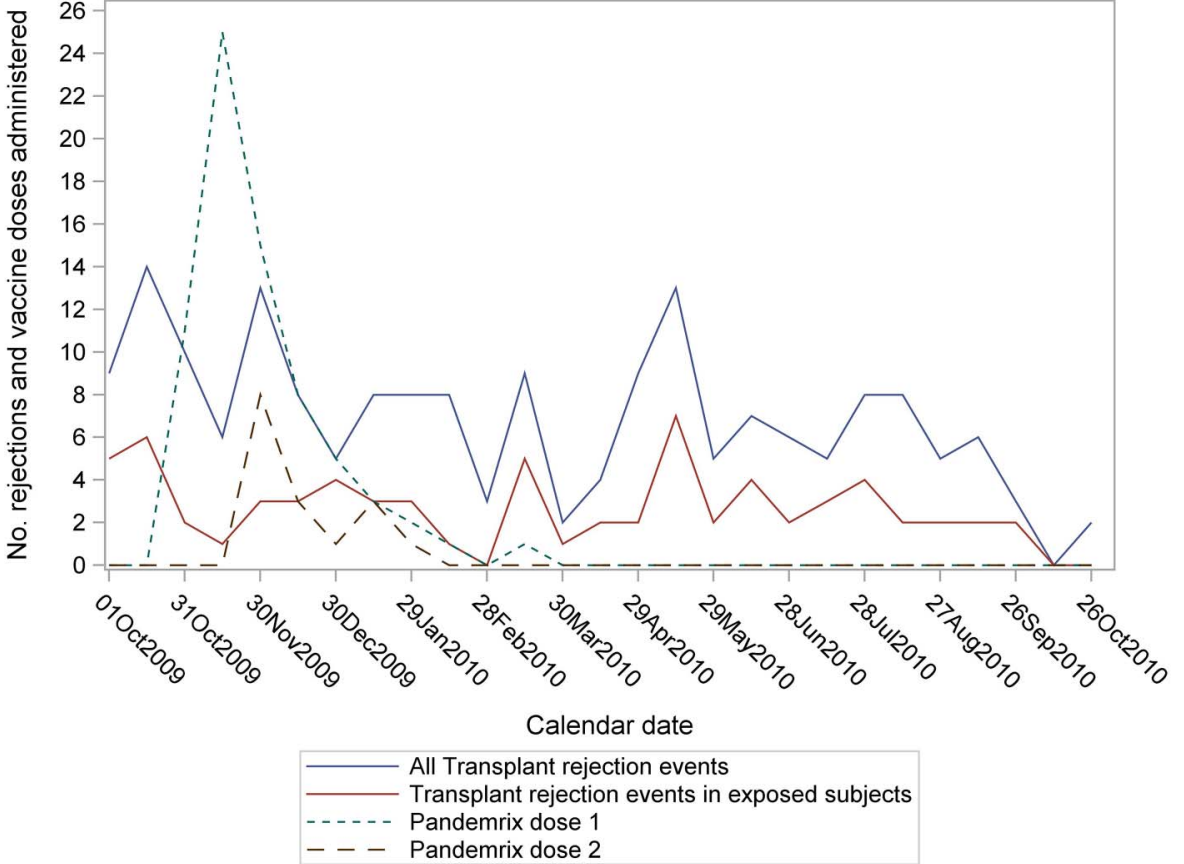

main analysis. A total of 72 exposed patients were included (only one additional participant was exposed after a subsequent SOT rejection event), of whom 11 had experienced a rejection in the 30 -day risk period, and 18 in the 60-day risk period among 73 exposed patients. The RI adjusted for time since transplantation was 1.11 (95\% CI 0.59 to 2.09 ) within 30 days after vaccination. When analyses were restricted to the 82 patients without previous SOT rejections, including 62 exposed patients, the RI within 30 days after Pandemrix vaccination was 1.02 (95\% CI 0.48 to 2.13 ).

The risk of rejection by organ could be assessed only for the kidney in 97 participants, including 53 exposed. The RI adjusted for time since transplantation was 0.85 (95\% CI 0.38 to 1.90 ) and 0.68 (95\% CI 0.33 to 1.40 ) within 30 and 60 days after vaccination, respectively. The
Figure $4 \mathrm{RI}$ of SOT rejection 30 and 60 days after vaccination with Pandemrix, for all organs pooled, adjusted for time since transplantation and vaccination with a TIV, opportunistic infections, malignancies/cancer (separately and in the same model). SOT, solid organ transplant; RI, relative incidence; Ntot, total number of participants; Nexp, number of exposed participants; TST, time since transplantation; SCCS, self-controlled case series. Seasonal influenza vaccine: vaccination with a seasonal trivalent influenza virus vaccine (TIV), any brand. Opportunistic infections: Cytomegalovirus, Herpes simplex, Varicella zoster, Epstein-Barr virus, Pneumocystis, BK virus, Aspergillus,

Cryptococcus, Listeria, Nocardia, Toxoplasma, Strongyloides, Leishmania, Trypanosoma, Candida.

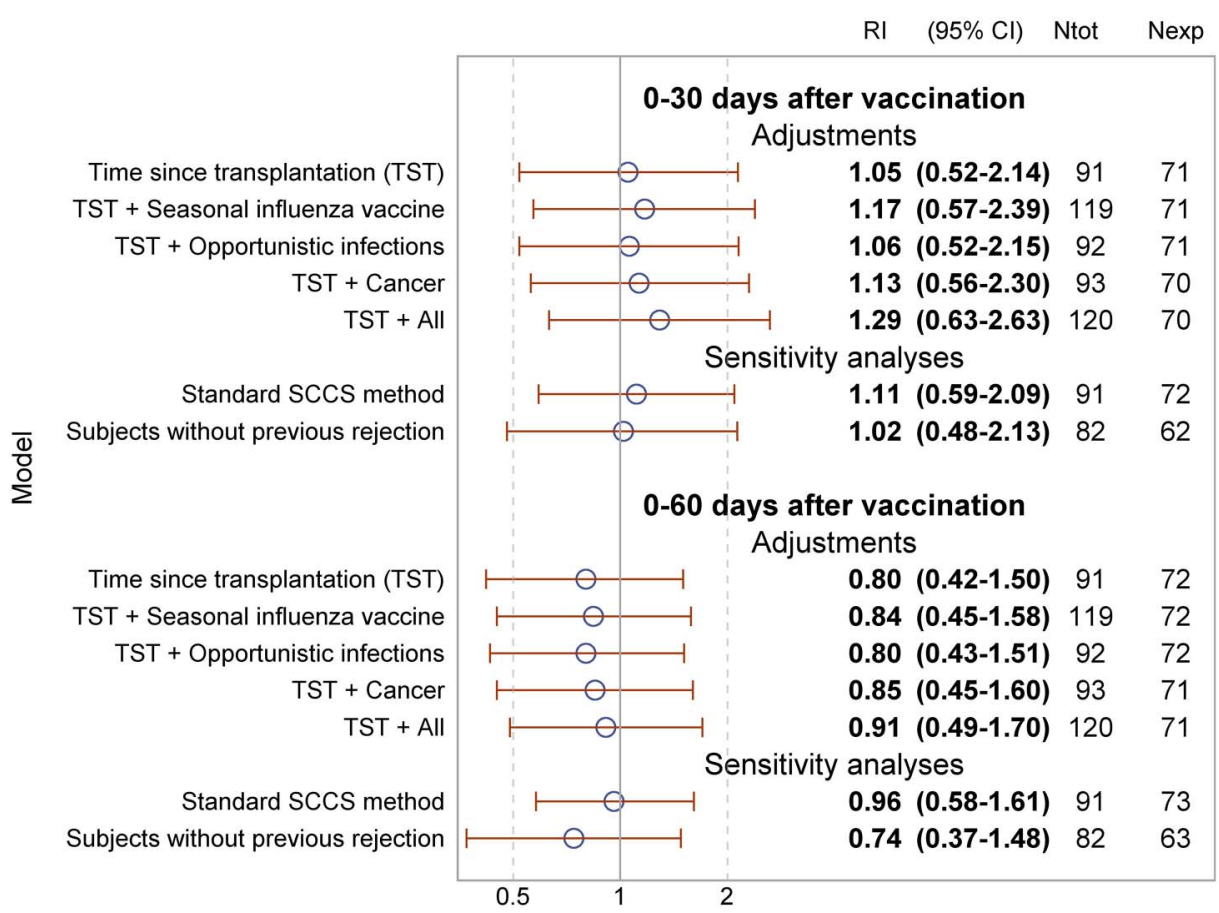


RI within 30 days after vaccination was $1.00 \quad(95 \%$ CI 0.44 to 2.25 ) in the model adjusted for TIV administration and malignancies/cancers (information for other covariates was not available).

\section{DISCUSSION}

\section{Principal findings}

To the best of our knowledge, this is the first study to formally assess the association between an H1N1 pandemic influenza vaccine and SOT rejection. It was designed to investigate a safety signal that emerged from routine pharmacovigilance and from published case series of SOT rejection after the 2009 H1N1 influenza pandemic mass vaccination campaign. Given the urgency for a rapid evaluation of the safety signal and the absence of vaccination after the end of the 2009/ 2010 pandemic season, a formal retrospective pharmacoepidemiology study in the CPRD database was the timeliest option to capture data from a large population while reflecting standard practice with respect to the management of transplanted patients.

The primary risk estimate is an RI of $1.05(95 \%$ CI 0.52 to 2.14) within 30 days after administration of Pandemrix. There is a consistent inference across sensitivity analyses, with point estimates around 1.0 and no upper $95 \%$ confidence limits higher than 2.3. None of the analyses show a statistically significant increased risk of SOT rejection following vaccination with Pandemrix.

\section{Strengths and weaknesses of our study}

Given the acuity, severity and clinical complexity of SOT rejection, transplanted patients are largely managed in specialty hospital settings. The HES inpatient database contains information for all NHS hospitals in England, which maximised the likelihood of capturing cases of SOT rejection. Diagnosis data are based on standardised coding practices, ensuring consistent information across the study population. The high proportion of participants in the study population with an HES link to the CPRD (93\%) confirmed the adequacy of using the HES as a primary reference data source for case finding. The CPRD, which was used to capture vaccine exposure and covariate data, has been extensively used in pharmacoepidemiology studies. ${ }^{28}$

The SCCS was primarily designed to study the association between acute events and transient exposures, and is well suited for the study of adverse reactions to vaccination. ${ }^{36} 40-45$ It handles fixed measured and unmeasured confounding, implicitly controlling for confounders such as socioeconomic status, access to healthcare, healthcare-seeking behaviour, confounding by indication, frailty, underlying chronic conditions and, in this study, type of transplanted organ and compliance with an immunosuppressive regimen. In the conventional SCCS, the propensity to be exposed is assumed to be independent of previous events (disease occurrence), whereas the modified SCCS requires to have only one event per participant and to censor each participant at the second event. Completeness and precision of exposure data for pandemic vaccination in the CPRD, and reliability of outcome data in the HES inpatient database (since based on clinical procedure codes) are crucial requirements for the SCCS design. To the best of our knowledge, relatively few vaccine safety studies report the use of the modified SCCS; ${ }^{4046} 47$ we therefore conducted a sensitivity analysis using the standard SCCS, which yielded consistent risk estimates.

On the basis of the assumption that time since transplantation is a risk factor for SOT rejection, the a priori decision was made to adjust all SCCS models for this variable. Interestingly, the majority of exposed participants $(63 / 71(89 \%))$ had been transplanted $>180$ days prior to the beginning of the study period, suggesting that time since transplantation is not a confounding factor in the present analysis, and limiting the opportunity to assess the potential confounding effect of time since transplantation. Nonetheless, as expected, all models showed consistent decreases in the risk of rejection with increasing time since transplantation (data not shown). With regard to potential confounding by TIV vaccination, adjusted analyses showed no differences from the primary risk estimates. Since most participants did not receive Pandemrix and a TIV on the same day, and there was limited overlap between risk periods for the two vaccines for most participants, these analyses allowed an independent estimation of the risk associated with Pandemrix and the adjustment for the potential confounding effect of TIV vaccination. Finally, the consistent range of risk estimates and CIs, all including 1.0 with upper $95 \%$ confidence limits around 2.0, suggests that the study was sufficiently powered to detect an association between Pandemrix and SOT rejection.

As with any retrospective observational study in a large healthcare database, this analysis has several limitations. First, SOT rejection is a complex clinical entity for which the likelihood of occurrence is affected by multiple risk factors such as viral (including H1N1 influenza virus) and bacterial infections, underlying medical conditions, medical history of the transplant recipient, and compliance with immunosuppressive treatment. These factors were not captured in these settings or had limited record completion in the CPRD. Thus, confounding by these unmeasured factors could not be fully evaluated; however, it is unlikely to have introduced systematic error into the analyses, and the SCCS design might have partially mitigated against some of these potential confounders, given their fixed nature. Another potential confounding factor is history of a previous rejection; the large majority of patients $(82 / 91(90.1 \%))$ had no rejection in the 6 months prior to the study period. The risk of rejection might also depend on the number of vaccine doses received; the number of patients having received two doses of Pandemrix was low $(\mathrm{N}=17)$ and limited the possibility of reliably assessing the effect of the second dose. Some studies indicate that risk factors for SOT rejection and the magnitude of the risk might 
vary by organ type ${ }^{48}$; however, given the small number of cases, the risk of rejection by organ could only be assessed for the kidney. Nonetheless, these estimates are informative, given that the kidney is the most commonly transplanted organ. ${ }^{49}$

Finally, the optimal duration of the risk period for SOT rejection following immunisation, if any, is unknown, and it is difficult to know whether a potential increased risk returns to baseline in the post-exposure control period as rejection-specific antibodies of unclear clinical significance might persist for a longer period of time. However, no significantly increased risk is observed using a 30-day or 60-day risk period.

Although standards of care of transplanted patients might vary across the country, documentation/coding guidelines in HES are standardised, thus limiting the variability in information at patient level. Given that study subjects are from databases representative of the general population, ${ }^{28} 30{ }^{32}$ results from this study may be extrapolated to the overall population of SOT recipients in England.

\section{Strengths and weaknesses in relation to other studies}

To the best of our knowledge, this SCCS analysis is the first pharmacoepidemiology study formally assessing the risk of SOT rejection following vaccination with an adjuvanted H1N1 2009 pandemic influenza vaccine. The results do not suggest an increased risk of rejection following administration of Pandemrix, and are in line with most descriptive clinical case series that have shown no increased risk of allograft dysfunction or clinical rejection in SOT recipients following H1N1 pandemic influenza vaccination. ${ }^{2}$ 15-23 50

\section{Meaning of the study}

Influenza A/H1N1 2009 infection caused substantial morbidity and mortality in SOT recipients worldwide. ${ }^{251-55} \mathrm{In}$ the largest study, involving 237 cases of medically attended H1N1 influenza infections from 26 transplant centres in the USA, infections resulted in hospitalisation in $71 \%$, admission to an intensive care unit in $16 \%$, and death in $4 \%$ of cases. ${ }^{54}$ Allograft dysfunction and clinical rejection were also reported in SOT recipients following pandemic influenza A/H1N1 infection. ${ }^{2}$ 51-53 5657 Pandemic influenza vaccination is therefore an important preventive measure in this medically vulnerable population at high risk of complications, and is considered clinically safe and well tolerated despite relatively modest efficacy. ${ }^{2} 5859$ In addition to investigating a safety signal, this study addresses the need for a formal pharmacoepidemiological analysis of the risk of SOT rejection in a large population of transplanted patients.

\section{CONCLUSIONS}

The results of this study are robust across a variety of sensitivity analyses and do not suggest an increased risk of SOT rejection following vaccination with Pandemrix.
This report also intends to bring some insight into the debate on the safety of influenza vaccination in transplant recipients, and to inform the benefit-risk evaluation of AS03-adjuvanted pandemic influenza vaccines in this patient population that is likely to remain a highrisk priority group for immunisation with future pandemic influenza vaccines.

Author affiliations

${ }^{1}$ Global Epidemiology, GSK Vaccines, Wavre, Belgium

${ }^{2}$ Business \& Decision Life Sciences, Brussels, Belgium (on behalf of GSK Vaccines)

${ }^{3}$ Department of R\&D, GSK, Uxbridge, Middlesex, UK

Acknowledgements The authors thank Morgane Guennec, Corinne Willame and Aurélie Le Plain for their contribution to the statistical analyses, and Jacques Bruhwyler, Robert Gardner and Mark Franco for writing support of the protocol and clinical study report. They also thank Shirin Khalili and Vincent Laporte (XPE Pharma and Science, and Business \& Decision Life Sciences, respectively, on behalf of GSK Vaccines) for publication management and Claire Verbelen (XPE Pharma and Science) for initial drafting of the manuscript and formatting of the final version.

Contributors CC, DR, FH, GDS and VS participated in the conception and design of the study. CC, DR, DW, FH, GDS, JL and VS assessed the feasibility of the study in CPRD/HES. FH assembled the data set and analysed the data with support from DR and GLCF. DW and JL provided CPRD/HES expertise. All authors contributed to the interpretation of the results. CC and FH wrote the manuscript. All authors had full access to the data (including statistical reports and tables), reviewed the manuscript, gave final approval before submission, and can take responsibility for the accuracy of the data analysis and the integrity of the results. CC is the guarantor.

Funding GlaxoSmithKline Biologicals SA was the funding source, and was involved in all stages of study design, conduct and analysis. It also took responsibility for all costs associated with the development and publishing of the present manuscript.

Competing interests $\mathrm{CC}, \mathrm{FH}, \mathrm{JL}, \mathrm{RD}$ and DW are employees of the GSK group of companies. GF and VS were employed by the GSK group of companies when the study was conducted. CC, JL, RD, DW and VS report ownership of stocks, stock options and/or restricted shares of the GSK group of companies. GDS's employer receives consulting fees from the GSK group of companies for the work submitted for publication and for other projects.

Ethics approval The study was approved by the Independent Scientific Advisory Committee (ISAC) of the CPRD, and was conducted in accordance with applicable regulatory requirements, the Guidelines for Good Pharmacoepidemiology Practices (GPP), applicable subject privacy requirements, and the guiding principles of the Declaration of Helsinki. It has been registered at http://www. clinicaltrials.gov (NCT01715792) and on the ENCePP e-register of studies (http:// www.encepp.eu/encepp/viewResource.htm?id=7070). A summary of the protocol is available at $h$ ttp://www.gsk-clinicalstudyregister.com (GSK study ID 116602). Code lists of ICD10, OPCS, READ and product codes are available in online supplementary file or online at ClinicalCodes.org.

Provenance and peer review Not commissioned; externally peer reviewed.

Data sharing statement Code lists of ICD10, OPCS, READ and product codes are available in online supplementary tables and on ClinicalCodes.org. The authors agree to make the study protocol, case definition algorithms and data extraction and analysis programmes available to the scientific community on request.

Open Access This is an Open Access article distributed in accordance with the Creative Commons Attribution Non Commercial (CC BY-NC 4.0) license, which permits others to distribute, remix, adapt, build upon this work noncommercially, and license their derivative works on different terms, provided the original work is properly cited and the use is non-commercial. See: http:// creativecommons.org/licenses/by-nc/4.0/ 


\section{REFERENCES}

1. Avery RK. Influenza vaccines in the setting of solid-organ transplantation: are they safe? Curr Opin Infect Dis 2012;25:464-8.

2. Cordero $\mathrm{E}$, Manuel $\mathrm{O}$. Influenza vaccination in solid-organ transplant recipients. Curr Opin Organ Transplant 2012;17:601-8.

3. Kumar D, Blumberg EA, Danziger-Isakov L, et al. Influenza vaccination in the organ transplant recipient: review and summary recommendations. Am J Transplant 2011;11:2020-30.

4. Zbinden $\mathrm{D}$, Manuel $\mathrm{O}$. Influenza vaccination in immunocompromised patients: efficacy and safety. Immunotherapy 2014;6:131-9.

5. Brakemeier $\mathrm{S}$, Schweiger $\mathrm{B}$, Lachmann $\mathrm{N}$, et al. Immune response to an adjuvanted influenza $\mathrm{A} \mathrm{H1N1}$ vaccine (Pandemrix®) in renal transplant recipients. Nephrol Dial Transplant 2012;27:423-8.

6. Katerinis I, Hadaya K, Duquesnoy R, et al. De novo anti-HLA antibody after pandemic $\mathrm{H} 1 \mathrm{~N} 1$ and seasonal influenza immunization in kidney transplant recipients. Am J Transplant 2011;11:1727-33.

7. Schaffer SA, Husain S, Delgado DH, et al. Impact of adjuvanted $\mathrm{H} 1 \mathrm{~N} 1$ vaccine on cell-mediated rejection in heart transplant recipients. Am J Transplant 2011;11:2751-4.

8. Danziger-Isakov L, Cherkassky L, Siegel H, et al. Effects of influenza immunization on humoral and cellular alloreactivity in humans. Transplantation 2010;89:838-44.

9. Burbach $\mathrm{G}$, Bienzle $\mathrm{U}$, Stark $\mathrm{K}$, et al. Influenza vaccination in liver transplant recipients. Transplantation 1999;67:753-5.

10. Candon $\mathrm{S}$, Thervet $\mathrm{E}$, Lebon $\mathrm{P}$, et al. Humoral and cellular immune responses after influenza vaccination in kidney transplant recipients. Am J Transplant 2009;9:2346-54.

11. Hurst FP, Lee JJ, Jindal RM, et al. Outcomes associated with influenza vaccination in the first year after kidney transplantation. Clin J Am Soc Nephrol 2011;6:1192-7.

12. Lawal A, Basler C, Branch A, et al. Influenza vaccination in orthotopic liver transplant recipients: absence of post administration ALT elevation. Am J Transplant 2004;4:1805-9.

13. Magnani G, Falchetti E, Pollini G, et al. Safety and efficacy of two types of influenza vaccination in heart transplant recipients: a prospective randomised controlled study. J Heart Lung Transplant 2005;24:588-92.

14. Scharpe J, Evenepoel $P$, Maes B, et al. Influenza vaccination is efficacious and safe in renal transplant recipients. Am J Transplant 2008;8:332-7.

15. Altamirano-Diaz L, West L, Humar A, et al. Early post-transplant vaccination with pandemic influenza $A / H 1 N 1$ vaccine in pediatric heart transplant recipients. Pediatr Transplant 2011;15:172-5.

16. Broeders NE, Hombrouck A, Lemy A, et al. Influenza A/H1N1 vaccine in patients treated by kidney transplant or dialysis: a cohort study. Clin J Am Soc Nephrol 2011;6:2573-8.

17. Crespo M, Collado S, Mir M, et al. Efficacy of influenza A H1N1/ 2009 vaccine in hemodialysis and kidney transplant patients. Clin J Am Soc Nephrol 2011;6:2208-14.

18. Felldin M, Studahl M, Svennerholm B, et al. The antibody response to pandemic H1N1 2009 influenza vaccine in adult organ transplant patients. Transpl Int 2012;25:166-71.

19. Goldschmidt I, Pfister ED, Becker M, et al. Acceptance and adverse events of the $2009 \mathrm{H} 1 \mathrm{~N} 1$ vaccination in immunosuppressed pediatric liver transplant recipients. J Pediatr 2011;158:329-33.

20. Meyer S, Adam M, Schweiger B, et al. Antibody response after a single dose of an AS03-adjuvanted split-virion influenza $A(H 1 N 1)$ vaccine in heart transplant recipients. Transplantation 2011;91:1031-5.

21. Salles MJC, Sens YAS, Malafronte $P$, et al. Antibody response to the non-adjuvanted and adjuvanted influenza A H1N1/09 monovalent vaccines in renal transplant recipients. Transpl Infect Dis 2012;14:564-74.

22. Schuurmans MM, Tini GM, Dalar L, et al. Pandemic 2009 H1N1 influenza virus vaccination in lung transplant recipients: coverage, safety and clinical effectiveness in the Zurich cohort. J Heart Lung Transplant 2011;30:685-90.

23. Siegrist CA, Ambrosioni J, Bel M, et al. Responses of solid organ transplant recipients to the AS03-adjuvanted pandemic influenza vaccine. Antivir Ther (Lond) 2012;17:893-903.

24. Torii $\mathrm{Y}$, Kimura $\mathrm{H}$, Ochi $\mathrm{N}$, et al. Immunogenicity of inactivated 2009 $\mathrm{H} 1 \mathrm{~N} 1$ influenza vaccine in pediatric liver transplant recipients. Vaccine 2011;29:4187-9.

25. Vazquez-Alvarez Mdel C, Medrano-Lopez C, Camino-Lopez M. $\mathrm{H} 1 \mathrm{~N} 1$ influenza vaccination and infection in pediatric heart transplants. J Heart Lung Transplant 2010;29:1318.

26. Hardelid P, Fleming DM, McMenamin J, et al. Effectiveness of pandemic and seasonal influenza vaccine in preventing pandemic influenza $\mathrm{A}(\mathrm{H} 1 \mathrm{~N} 1) 2009$ infection in England and Scotland 20092010. Euro Surveill 2011;16:pii: 19763.
27. Sethi M, Pebody R. Pandemic H1N1 (swine) influenza vaccine uptake amongst patient groups in primary care in England 2009/10. Departement of Health-Health Protection Agency, 2010. https:// www.gov.uk/government/uploads/system/uploads/attachment_data/ file/215977/dh 121014.pdf (accessed 27 Feb 2015).

28. Herrett E, Gallagher AM, Bhaskaran K, et al. Data resource profile: Clinical Practice Research Datalink (CPRD). Int J Epidemiol 2015;44:827-36.

29. Herrett E, Thomas SL, Schoonen WM, et al. Validation and validity of diagnoses in the General Practice Research Database: a systematic review. Br J Clin Pharmacol 2010;69:4-14.

30. Williams T, van Staa T, Puri S, et al. Recent advances in the utility and use of the General Practice Research Database as an example of a UK Primary Care Data resource. Ther Adv Drug Saf 2012;3:89-99.

31. Health and Social Care Information Centre. Hospital Episode Statistics. http://www.hscic.gov.uk/hes (accessed 27 Feb 2015).

32. Sinha S, Peach G, Poloniecki JD, et al. Studies using English administrative data (Hospital Episode Statistics) to assess health-care outcomes-systematic review and recommendations for reporting. Eur J Public Health 2013;23:86-92.

33. Department of Health/Health Protection Agency. Pandemic H1N1 (swine) influenza vaccine uptake amongst patient groups in primary care in England 2009/10. http://www.dh.gov.uk/prod_consum_dh/ groups/dh digitalassets/@dh/@en/@ps/documents/digitalasset/dh 121014.pdf (accessed 27 Jan 2014).

34. Department of Health, United Kingdom. Pandemic H1N1 Vaccine Uptake Figures for England by SHA and PCT. http://webarchive. nationalarchives.gov.uk/20130107105354/http:/www.dh.gov.uk/en/ Publicationsandstatistics/Publications/PublicationsPolicy AndGuidance/DH_114203 (accessed 25 Mar 2015).

35. Weldeselassie $Y G$, Whitaker HJ, Farrington CP. Use of the self-controlled case-series method in vaccine safety studies: review and recommendations for best practice. Epidemiol Infect 2011;139:1805-17.

36. Whitaker HJ, Farrington $\mathrm{CP}$, Spiessens B, et al. Tutorial in biostatistics: the self-controlled case series method. Stat Med 2006;25:1768-97.

37. Farrington $\mathrm{CP}$, Whitaker $\mathrm{HJ}$, Hocine MN. Case series analysis for censored, perturbed, or curtailed post-event exposures. Biostatistics 2009;10:3-16.

38. Farrington CP. Relative incidence estimation from case series for vaccine safety evaluation. Biometrics 1995;51:228-35.

39. SAS Institute. Editorial Guidelines. http://www.sas.com/en_us/legal/ editorial-guidelines.html (accessed 25 Mar 2015).

40. Andrews N, Stowe J, Al-Shahi Salman R, et al. Guillain-Barre syndrome and $\mathrm{H} 1 \mathrm{~N} 1$ (2009) pandemic influenza vaccination using an AS03 adjuvanted vaccine in the United Kingdom: self-controlled case series. Vaccine 2011;29:7878-82.

41. Arnheim-Dahlstrom L, Hallgren J, Weibull CE, et al. Risk of presentation to hospital with epileptic seizures after vaccination with monovalent AS03 adjuvanted pandemic A/H1N1 2009 influenza vaccine (Pandemrix): self controlled case series study. BMJ 2012;345:e7594.

42. Donegan K, Beau-Lejdstrom R, King B, et al. Bivalent human papillomavirus vaccine and the risk of fatigue syndromes in girls in the UK. Vaccine 2013;31:4961-7.

43. Musonda P. The self-controlled case series method: performance and design in studies of vaccine safety [PhD thesis]. The Open University, 2006.

44. Romio S, Weibel D, Dieleman JP, et al. Guillain-Barre syndrome and adjuvanted pandemic influenza $A$ (H1N1) 2009 vaccines: a multinational self-controlled case series in Europe. PLOS ONE 2014;9:e82222.

45. Warren-Gash $\mathrm{C}$, Hayward AC, Hemingway $\mathrm{H}$, et al. Influenza infection and risk of acute myocardial infarction in England and Wales: a CALIBER self-controlled case series study. J Infect Dis 2012;206:1652-9.

46. Crawford NW, Cheng A, Andrews N, et al. Guillain-Barre syndrome following pandemic (H1N1) 2009 influenza A immunisation in Victoria: a self-controlled case series. Med J Aust 2012;197:574-8.

47. Yung CF, Chan SP, Soh S, et al. Intussusception and monovalent rotavirus vaccination in Singapore: self-controlled case series and risk-benefit study. J Pediatr 2015;167:163-8.el.

48. Klein A, Lewis C, Madsen J. Organ Transplant. A Clinical Guide, 2011, Cambridge University Press, Section 2 (p63-69), Section 3 (p122-127), Section 4 (p173-181), Section 5 (p231-237) and Section 6 (p286-294). 
49. European Society for Organ Transplantation. http://www.esot.org/ ESOT/home (accessed 25 Mar 2015).

50. Läkemedelsverket Medical Products Agency. Final summary of adverse drug reaction reports in Sweden with Pandemrix through October 2009 -mid April 2010. 2010. http://www.lakemedelsverket. se/upload/eng-mpa-se/Pandemrix\%20ADRs\%20in\%20Sweden\% 2015\%20april\%202010.pdf (accessed 27 Feb 2015)

51. Crockett F, Mal H, Amazzough K, et al. H1N1 (2009) influenza A infection in transplant recipient patients: a comparative study versus non-transplanted patients. Rev Pneumol Clin 2011;67:199-208.

52. Fox BD, Raviv $\mathrm{Y}$, Rozengarten $\mathrm{D}$, et al. Pandemic influenza (H1N1) impact on lung transplant recipients and candidates. J Heart Lung Transplant 2010;29:1034-8.

53. Freitas TV, Ono G, Correa $\mathrm{L}$, et al. Clinical manifestations and evolution of infection by influenza A (H1N1) in kidney transplant recipients. J Bras Nefrol 2011;33:136-41.
54. Kumar D, Michaels MG, Morris MI, et al. Outcomes from pandemic influenza A H1N1 infection in recipients of solid-organ transplants: a multicentre cohort study. Lancet Infect Dis 2010;10:521-6.

55. Low CY, Kee T, Chan KP, et al. Pandemic (H1N1) 2009 infection in adult solid organ transplant recipients in Singapore. Transplantation 2010;90:1016-21.

56. Vistoli F, Focosi $\mathrm{D}$, De Donno $\mathrm{M}$, et al. Pancreas rejection after pandemic influenza virus $\mathrm{A}(\mathrm{H} 1 \mathrm{~N} 1)$ vaccination or infection: a report of two cases. Transpl Int 2011;24:e28-9.

57. Stucchi RS, Boin IF, Angerami RN, et al. Correlations between A/ $\mathrm{H} 1 \mathrm{~N} 1$ influenza and acute cellular rejection in liver transplantation patients. Transplant Proc 2010:42:4184-6.

58. Beck CR, McKenzie BC, Hashim AB, et al. Influenza vaccination for immunocompromised patients: systematic review and meta-analysis by etiology. J Infect Dis 2012;206:1250-9.

59. Pittet LF, Posfay-Barbe KM. Immunization in transplantation: review of the recent literature. Curr Opin Organ Transplant 2013;18:543-8. 